\title{
Fluorine F 18-Active Site Inhibited Factor VIIa
}

National Cancer Institute

\section{Source}

National Cancer Institute. Fluorine F 18-Active Site Inhibited Factor VIIa. NCI Thesaurus.

Code C157486.

A radioconjug ate containing a tissue factor (TF) tracer, a derivative of activated factor VII (FVIIa), labeled with fluorine F 18, with potential use for TF-expressing tumor imaging using positron emission tomography (PET). Upon administration of fluorine $\mathrm{F} 18$ active site inhibited factor VIIa (18F-ASIS), the active site inhibited factor VIIa (FVIIai) moiety selectively targets and binds, with a much higher binding affinity than FVIIa, to TF. Upon PET imaging, tumor cells expressing TF can be visualized and assessed. TF, a transmembrane glycoprotein, is overexpressed on certain tumor cell types and is correlated with tumor aggression and poor prognosis. 